\title{
SOHO and Cluster
}

\section{SOLAR AND GEOSPHERIC PLASMAS AND SOLAR STRUCTURE}

\author{
V. Domingo and R. Schmidt
}

\author{
Space Science Department of ESA \\ European Space Technology and Research Centre \\ Noordwijk, The Netherlands
}

\begin{abstract}
Understanding plasma phenomena arising in the Sun and in planetary magnetospheres is essential for establishing the effect of the Sun on the Earth. Moreover, the Earth's magnetosphere and the solar atmosphere are the only large scale "laboratories without windows" where dynamical phenomena in plasmas with a very large range of densities, temperatures, compositions, etc. can be realistically studied, either in situ in planetary magnetospheres or using remote sensing plasma diagnostics. Finally, as solar plasma phenomena are powered by processes arising inside the Sun, it is also necessary to link observations with those relating to the Sun's interior.
\end{abstract}

Important questions relating to solar and geospheric plasmas and solar structure will be addressed by the Solar-Terrestrial Science Programme - a joint European Space Agency (ESA)/ NASA undertaking comprising the $\mathrm{SOHO}$ and Cluster satellite missions. SOHO, the Solar and Heliospheric Observatory, is a single spacecraft that will carry helioseismology sensors, extreme ultraviolet telescopes and spectrometers, coronagraphs, and solar wind composition analysers. Cluster comprises a set of four spacecraft, equipped to measure fields and flows in three dimensions, to be flown in a "cluster" formation through the interesting regions of the geosphere. Both missions are being developed for launch in the second half of 1995.

The programme as a whole aims to study:

- the structure of the solar interior and its dynamics:

- the physics of the plasma phenomena that take place in the solar atmosphere,

Vicente Domingo is a SOHO Project Scientist in the Solar and Heliospheric Science Division at ESA's Space Science Department, ESTEC, NL-2200 AG Noordwijk. He studied in Spain and worked on cosmic ray research before developing solar physics experiments.

Rudolf Schmidt joined ESA's Space Science Department's Planetary Science Division as a Project Scientist in 1982 and worked on Spacelab's magnetometer experiment. He received a Ph.D a few years before from the University of Graz, Austria, where he was involved with a space physics experiment. heat the solar corona and give rise to the solar wind;

- "in situ" and in detail the small scale, plasma physics processes which take place at key points of the terrestrial magnetosphere, such as the cusps, plasma sheets, etc., where active plasma phenomena arise, notably energy transfer from the solar wind and reconnection of magnetic field lines.

This short review of the overall programme will outline the physics objectives and methodologies; the projects themselves are summarised on page 215 .

\section{SOHO - The Solar and Helioscopic Observatory}

The space-based solar mission $\mathrm{SOHO}$ (Fig. 1) has been designed with two solar physics objectives in mind: on the one hand to investigate the structure and dynamics of the solar interior using helioseismology and by measuring solar irradiance variations; and on the other hand, to achieve a deeper understanding of the physical processes that form and heat the solar corona, maintain it and give rise to the expanding solar wind. These latter goals will be addressed by means of imaging and spectroscopic plasma diagnostics of the chromosphere and of the transition and corona regions of the Sun, and by in situ measurements of the solar wind.

\section{The Sun's interior}

A Fourier analysis of the motion of the solar surface in space and in time has revealed the presence of a very large number of individual mo-

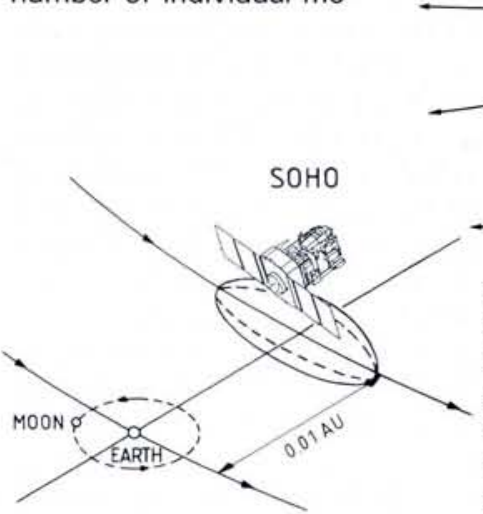

des of oscillation. Their periods range from a few minutes to several hours, and horizontal wavelengths range from the solar circumference to a few thousand kilometres. The modes are coherent over at least a day, and in many cases much longer. Their frequencies can thus be determined with very high accuracy, and this makes them sensitive probes of the solar interior.

Helioseismological investigations have already produced significant results, such as a determination of the equatorial differential rotation rate over part of the solar interior. They show that by obtaining a more complete coverage of the spectrum of oscillations, in both spatial and temporal scales, one should be able to achieve a major breakthrough in our understanding of the solar interior. We presently have a reasonable overall analysis which is lacking in important details such as the degree of mixing and the structure of the magnetic fields. Several coordinated efforts have thus been initiated to study solar oscillations employing observatories located on Earth. However, it is known that significant parts of the oscillations spectrum cannot be measured from Earth with the required sensitivity owing to the "twinkling" of bright spots, noise introduced by the Earth's diurnal rotation and fluctuations in the transparency of its at- 
mosphere. A set of SOHO's helioseismological instruments will therefore address those parts of the solar oscillations spectrum that cannot be properly observed from the ground.

$\mathrm{SOHO}$ will perform long and uninterrupted series of oscillation measurements of the full solar disk, in both the velocity and the irradiance domains: in this way information will be obtained about the low degree modes of oscillation caused by waves that concentrate in the solar nucleus. SOHO will also measure oscillations on the surface of the Sun with high angular resolution $\left(1.4 \times 1.4 \operatorname{arcsec}^{2}\right)$. This will permit a determination of the frequency spectra of many different resonant waves with many oscillation modes, including waves of very short spatial wavelengths carrying extremely precise information about the Sun's convection zone - the thin, outer layer of the solar interior.

\section{The corona and its expansion}

The hot solar atmosphere or corona is a fascinating region of the Sun as it comprises magnetised plasma whose origin, dynamics and structure are poorly understood. Most of the phenomenological knowledge we posses about the corona was obtained in the 1970's by satellite observatories, in particular, the Apollo Telescope Mount in NASA's Skylab. The study of such information shows that understanding of the physical processes that form the corona requires a coordinated investigation, with the appropriate spatial and time resolutions, of the physical parameters of the underlying structures. These parameters include the composition, density, temperatures, temperature and density gradients, velocities, mass flows, magnetic field, the evolution of the magnetic structures, shocks, waves, etc. - all of which need to be measured at, for instance, coronal holes (regions of lower temperature and intensity where the magnetic field is open), in magnetic loops and at different heights if one wants to test existing magneto-hydrodynamic models of the coronal structures, and to develop them further.

To undertake a coordinated investigation of the solar corona and its expansion into the solar wind, $\mathrm{SOHO}$ will carry:

1) A combination of telescopes, spectrometers and coronagraphs. These instruments will obtain plasma diagnostic information by analysing emission line spectra in the visible and extreme ultraviolet (EUV) parts of the spectrum, where ions of the hot solar atmosphere emit preferentially. For example, the electron density and temperature can be obtained from intensity ratios of line pairs emitted at conditions suitable for observation (e.g., adequate intensity, appropriate wavelength).

Line shifts and the broadening of lines can be used to derive line-of-sight velocity

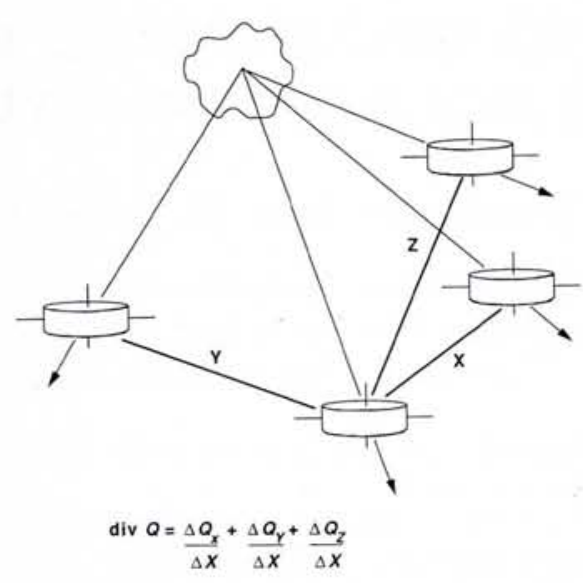

Fig. 2 - Simplified representation of the procedure for determining a vector quantity describing near-Earth space plasma using four spacecraft: the divergence of a quantity is computed from four non-coplanar measurements. It is essential to know the inter-spacecraft separation and the time of measurements to high accuracy.

fields and temperatures. The same instruments will provide information on the evolution of structures from the chromosphere out to the external corona.

2) An array of in situ particle analysing detectors will provide information on the charge state and isotopic composition of ions in the solar wind, and on the charge and isotopic composition of energetic particles generated by the Sun.

\section{Cluster - Four Identical Spacecraft}

Cluster is a mission comprising four spacecraft to investigate, in three dimensions, small-scale plasma phenomena in the near-Earth space plasma. Single-spacecraft measurements in space are intrinsically unable to distinguish unambiguously between spatial and temporal variations in the ambient plasma. With two satellites, this ambiguity is removed only for simple motions of essentially onedimensional structures. An unambiguous determination of the shape and dynamics of three-dimensional structures requires, as a minimum, four spacecraft in a tetrahedral configuration and equipped with instruments which measure plasma fields and flows in three dimensions (Fig. 2). Accurate data interpretation also calls for identical instrumentation aboard each of the spacecraft and the capability to match the inter-spacecraft separation distance with the scale lengths typical of the plasma processes to be studied.

Cluster provides all these features for the first time and there are indications that other multi-spacecraft missions comprising four (or more) satellite may follow in the not-too-distant future. The mission has been conceived to investigate, in three dimensions, space plasma processes that are believed to be ubiquitous in the Universe but are most easily accessible in Earth's environment. The phenomena to be studied (e.g., the interaction of different plasmas, turbulence, etc.) involve smallscale plasma structures with spatial extensions ranging from a few to a few tens of Larmor radii - corresponding to several hundreds to several tens of thousands of kilometres. Cluster therefore requires a new space segment based upon four spacecraft in non-coplanar orbits with adjustable separation distances.

The most obvious applications of the four-spacecraft technique are determinations of vector quantities such as plasma current densities (from the curl of the magnetic field $B$ ), the vorticity of the plasma flow (from the curl of the velocity v), shear flows (from the divergence of the electric field $E$ ), and the momentum balance across plasma boundaries (e.g., from the divergence of magnetic stress and pressure tensors). The intrinsic assumption is that plasma conditions are reasonably stable over scale lengths corresponding to the inter-spacecraft separation distances. Consequently, an adjustment of the spacecraft separation according to the scale length of the plasma phenomena to be studied is an essential ingredient of the Cluster mission.

\section{Orbit and separation strategy}

Ariane V, a new, more powerful European launcher currently under development which is due to be launched for the first time in 1995, will take the four Cluster spacecraft into a near-ecliptic transfer orbit. Each will carry a propulsion system so that the four can reach the final polar orbit after series of manoeuvres that temporarily send them well beyond the orbit of the Moon. The final apogee and perigee are 24 and 4 Earth radii $\left(R_{\mathrm{E}}\right)$, respectively. Each will adopt slightly different orbits such that a near-tetrahedral arrangement of the spacecraft is established. After arrival in their orbits, the spacecraft will be commissioned, their booms and mechanisms deployed and instruments activated. The base length of the tetrahedron will be changed every six months to match the scale lengths of the plasma phenomena to be investigated. The current scenario calls for six monthly intervals with the following separations: 1000 $\mathrm{km}$, then $1 R_{\mathrm{E}^{\prime}}$ subsequently 200-500 $\mathrm{km}$, and finally $3 R_{\mathrm{E}}$.

\section{Complementary Activities}

Firm plans have been established to organise a ground-based observation programme in coordination with the Cluster mission so as to to tackle plasma phenomena at magnetically conjugate points in the ionosphere and in space. Cluster will lie on a given magnetic field line and it will 
be helpful to simultaneously monitor phenomena in regions where the same line re-enters the ionosphere. Ground-based ionospheric radar, balloons, sounding rockets, optical instruments, magnetometer chains, etc. located at high geographical latitudes (above $70^{\circ} \mathrm{N}$ ) will be the key elements of this cooperation .

Under the terms of a bilateral agreement with the European Space Agency, it is the intention of the Institute of Space Research, Moscow, to complement the Cluster mission by providing another spacecraft, thereby broadening the science scope and international collaboration. The hope is that the Soviet spacecraft, based on a novel design using a solar sail for stabilisation and propulsion, will be closely coordinated with Cluster in all aspects of the mission execution. The orbit will be very similar to the Cluster orbit, the respective Mission Control Centres will be connected via computer and voice links and joint scientific activities, including teleconferencing, are planned to maximise the output from the collaboration.

\section{Conclusions}

The $\mathrm{SOHO} /$ Cluster satellite missions scheduled for the second half of this decade will provide an unique opportunity to advance our understanding of the physics of the solar-terrestial system. The spacebased solar observatory $\mathrm{SOHO}$ will be the centrepiece of a coordinated study of the structure of the Sun's interior and the phenomena governing the thermal and dynamic structures of the solar atmosphere.

Coordination of the four Cluster spacecraft with other spacecraft and with ground-based geomagnetic projects will constitute the most ambitious programme so far to examine in detail plasma phenomena in the Earth's magnetosphere.

\section{SOHO and Cluster Missions}

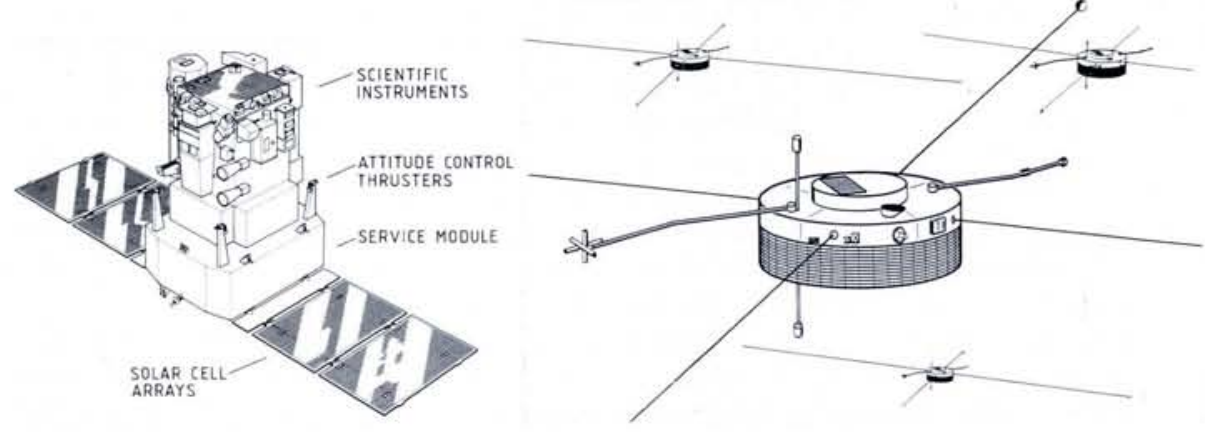

a, left) The SOHO spacecraft (3.8 $\mathrm{m}$ height, $9.5 \mathrm{~m}$ span) will be permanently pointed at the centre of the Sun, within 10 arcsec and with a stability better than 1 arcsec over 15 minutes. $b$, right) Cluster spacecraft: each can be represented as a cylinder, with the experiments distributed in a disc-like module, spinning at $15 \mathrm{rpm}$ for efficient stabilisation, to allow data taking in all directions and to keep extended the $50 \mathrm{~m}$ wire antennae for measuring electric fields. Note also the fluxgate and search coil magnetometers mounted at the tips of shorter, rigid booms.

\section{sоHO}

The SOHO spacecraft (see figure) consists of a platform that allows the solarpointing telescopes to observe the Sun or part of it with a stability of 1 arcsec per 15 minutes, and a module that carries services, including the power source (solar arrays) and systems for data handling, telemetry and pointing stabilisation. The total mass and available power will be about $1850 \mathrm{~kg}$ and $750 \mathrm{~W}$, respectively, of which approximately $640 \mathrm{~kg}$ and $450 \mathrm{~W}$ will be allocated to the payload.

Twelve teams of investigators, comprising about 200 individual scientists, and engineers from academic institutions from 15 countries, are developing the instruments and the necessary data handling and analysis tools, as well as preparing the theoretical framework.

\section{Operations}

It is planned to launch SOHO in July 1995 using an Atlas II AS rocket from the Kennedy Space Center in Florida. The space-

craft will be injected into a halo orbit around the LI Sun-Earth Lagrangian point, about $1.5 \times 10^{6} \mathrm{~km}$ Sun-ward from the Earth. Designed for a lifetime of two years, SOHO will be equipped, however, with sufficient on-board consumables for an additional four year's of operation.

Telemetric data from $\mathrm{SOHO}$ will be received by ground stations belonging to NASA's Deep Space Network. The scientific instruments alone generate about $200 \mathrm{kbits} / \mathrm{s}$ of data of which $165 \mathrm{kbits} / \mathrm{s}$ will be from the instrument for imaging solar oscillations.

An Experiment Operations Facility (EOF) located at NASA's Goddard Space Flight Center will be used to coordinate and plan the scientific operations of the payload, and will allow, in particular, real-time operation and control of the solar remote-sensing imaging and spectrometric instruments. It is planned to offer facilities for guest observers or investigators at the EOF while $\mathrm{SOHO}$ is operational.

Several $\mathrm{SOHO}$ investigation teams are planning to develop extensive data analysis facilities at their institutes, both in Europe and the USA. Moreover, a subsidiary EOF and data analysis centre in Europe is being considered, to facilitate the access by European scientists for $\mathrm{SOHO}$ operation and data analysis. All the operation and data centres will be connected electronically among themselves and with solar ground observatories, so that coordinated campaigns of operations, involving the study of solar phenomena occurring at many wavelengths, can be planned and executed.

\section{Cluster}

Eleven scientific instruments will be carried on each Cluster spacecraft (see figure). The total weight of these instruments per spacecraft is $72 \mathrm{~kg}$ and they consume in their standard mode of operation about $47 \mathrm{~W}$ of electrical power. The average data rate produced for transmission to a ground station amounts to $22 \mathrm{kbits} / \mathrm{s}$ and high speed modes to $250 \mathrm{kbits} / \mathrm{s}$ are foreseen.

\section{Cluster Science Data System}

Six major European research centres plan to link together their computers via the European Space Agency's ESANET network to support a rapid and efficient scientific interpretation of the data communicated by the Cluster spacecraft. Active cooperation is also planned with a number of non-ESA member states (e.g. USA, USSR, etc.). Two institutes from Hungary and China have submitted proposals for participation in the Cluster Science Data System and ways to cooperate with these institutes are presently being examined.

The data centres will produce and distribute the low and high resolution data plots: they will also maintain and distribute science-data access software and calibration files.

Scientists will be able to communicate electronically among themselves and with the Operations Control Centre located at the European Space Operations Centre in Darmstadt, Germany. During specific campaigns, this could take place in the framework of electronic conferencing: services such as data catalogues, bulletin boards, mail exchange, etc., will be incorporated. The final products of the scientific mission will be archived after the end of the space mission.

\section{Theory support}

The analysis of data measured simultaneously by the four spacecraft is quite complex. In order to support not only the data interpretation but also the preparation of the mission as such, a programme, using state-of-the-art supercomputers, has been started to develop numerical simulations of plasma processes of interest to the Cluster mission. Some 12 European groups have joined this "European Network for numerical simulations of plasma processes". The main objectives are to calculate signatures scale-lengths of plasma processes, and analyses for data interpretation. A very desirable outcome of this programme will be the training of students in using the most modern computers. 